TP Periodica Polytechnica Mechanical Engineering

62(3), pp. 203-208, 2018

https://doi.org/10.3311/PPme.11604

Creative Commons Attribution (i)

RESEARCH ARTICLE

\section{Weight Function Approach for Semi Elliptical Crack at Blade Mounting Locations in Steam Turbine Rotor System}

\author{
Damarla Kiran Prasad ${ }^{1 *}$, Kavuluri Venkata Ramana ${ }^{2}$, \\ Nalluri Mohan Rao ${ }^{3}$
}

Received 15 October 2017; accepted 13 March 2018

\begin{abstract}
This paper presents analysis of stress intensity factors at blade mounting locations of steam turbine rotor system. General expressions for the stresses induced in a rotating disc are derived and these equations are applied to steam turbine rotor disc. It is observed that the radial stress increases instantly at blade mounting location which indicates the probability of crack initiation and growth. A semi elliptical crack is considered at that location and weight function approach is used to determine the stress intensity factors. The results are validated with the influence coefficient approach. The differences of present approach with influence coefficient approach are less than $3 \%$. Hence the present approach is suitable for determination of stress intensity factors in a semi elliptical crack at blade mounting locations of a steam turbine rotor disc.
\end{abstract}

\section{Keywords}

blade mounting locations, turbine rotor, semi elliptical crack, weight function approach, influence coefficient approach

\footnotetext{
${ }^{1}$ Gudlavalleru Engineering College,

Gudlavalleru - 521356, India

${ }^{2} \mathrm{~K}$. L. University,

Vaddeswaram - 522502, India

${ }^{3}$ Jawaharlal Nehru Technological University Kakinada,

Kakinada - 533003, India

"Corresponding author, e-mail: damarlakiran@gmail.com
}

\section{Introduction}

Rotating components of steam turbine system like rotors and blades are most critical components as they are subjected to high mechanical and thermal loads which affect the integrity and life of the steam turbine system there by the entire plant. Blade mounting locations of a steam turbine rotor disc are subjected to complex loads due to the operating conditions of the rotor. Consequently, the blade mounting locations are the critical areas and most susceptible to the crack initiation. Failure of the rotors is evidenced by the high localized stresses induced at the blade attachment locations. Therefore prediction of stresses induced at those locations and the associated stress intensity factors of the cracks generated at blade mounting locations are very important in determining the likely crack growth to prevent the failures under normal operating conditions. A number of researchers have investigated the reasons for the failure of the rotor and discs. Barella et al. [1] investigated the rotor failure of $60 \mathrm{MW}$ unit of thermal power plant, conducted several types of examinations and identified that the stress field generated due to frequent startup cycles and blade fixing method are the root causes for the failure. Dianyin $\mathrm{Hu}$ et al. [2] proposed stochastic failure crack model to determine the probability of crack growth to achieve specific crack size and performed probabilistic analysis on crack growth. Hyojin Kim et al. [3] performed different types of analysis like Material composition analysis, hardness measurement and microstructure analysis to determine the cause of crack initiation and identified that crack initiated due to corrosion pits and propagated due to blade vibration. Qinkai Han et al. [4] proposed discrete state transition matrix to identify numerically the instability regions in an asymmetric disc of a rotor bearing system. Alegre and Cuesta [5] presented closed form solutions to determine the influence coefficients which can be subsequently used to determine the stress intensity factors at the surface and deepest points of a semi elliptical crack in pressure vessels. Shlyannikov et al. [6] identified that initiation and growth of crack in aircraft gas engine occurred at disc and blade attachment and proposed two and three dimensional finite element approach models to study the stress - strain state and stress intensity factors and also design modifications and repair 
technologies. Mazur and Hernandez-Rossette [7] investigated the failure of the two rotor discs and determined that the failure of the disc is because of the stress corrosion due to static stresses, humid operating conditions and material susceptibility and proposed welding repair procedure as a result of which the rotor disc were returned to service. Jianfu Hou et al. [8] examined the cracked disc and identified that the flaw was due to hammer forging and proposed advanced finite element analysis approach to analyze the turbine discs and predicted the fatigue crack growth. Pang and Reed [9] studied the effect of micro structure on crack initiation and crack growth in turbine disc and identified that the crack initiation was due to porosity on or near the surface and also due to grain boundary oxidization and suggested that large grain size improves the life time under the considered test conditions.

\section{Stresses at blade mounting locations of steam turbine rotor}

The differential equation (1) which represents a rotating disc subjected to non zero temperature gradient along the radius is

$$
\begin{aligned}
& \frac{d^{2} v}{d r^{2}}+\left(\frac{1}{t} \frac{d t}{d r}+\frac{1}{r}\right) \frac{d v}{d r}+\left(\frac{v}{t r} \frac{d t}{d r}-\frac{1}{r^{2}}\right) v \\
& +(1+\vartheta) \alpha\left(\frac{d T}{d r}+\frac{T}{t} \frac{d t}{d r}\right)+\left(1-\vartheta^{2}\right) \frac{\gamma \omega^{2} r}{E}=0 .
\end{aligned}
$$

Where $\mathrm{v}$ is the radial deflection, $\mathrm{r}$ is the radius of the rotating disc, $\mathrm{t}$ is the thickness of the disc, $\mathrm{T}$ is the temperature. For a disc of varying thickness, considering dimensionless radius parameter $\mathrm{k}=\mathrm{r} / \mathrm{R}$, the modified equation is

$$
\begin{aligned}
& k^{2}(1-k) \frac{d^{2} v}{d t^{2}}+k(1-2 k) \frac{d v}{d t}-(1-k+\vartheta k) v \\
& =-C R^{3} k^{3}+C R^{3} k^{4}
\end{aligned}
$$$$
\text { Where } C=\frac{\left(1-\vartheta^{2}\right) \gamma \omega^{2}}{E} \text {. }
$$

The solution for Eq. (2) consists of associated homogeneous equation solution and the particular integral and the radial and tangential stresses induced are given by

$$
\begin{aligned}
& \sigma_{r}=A a_{r}+B b_{r}+\sigma_{0} g_{r} \\
& \sigma_{t}=A a_{t}+B b_{t}+\sigma_{0} g_{t} .
\end{aligned}
$$

Where

$$
\begin{gathered}
a_{r}=\frac{d u_{1}}{d t}+\vartheta \frac{u_{1}}{t}, b_{r}=\frac{d u_{2}}{d t}+\vartheta \frac{u_{2}}{t} \\
a_{t}=\frac{u_{1}}{t}+\vartheta \frac{d u_{1}}{d t}, b_{t}=\frac{u_{2}}{t}+\vartheta \frac{d u_{2}}{d t} \\
g_{r}=-\frac{(3+\vartheta)}{(11+\vartheta)} k^{2}+\frac{(2+\vartheta)(3+\vartheta) k}{(5+\vartheta)(11+\vartheta)}-\frac{(3)(3+\vartheta) k}{(2+\vartheta)(3+\vartheta)}
\end{gathered}
$$

$$
g_{t}=-\frac{(1+3 \vartheta)}{(11+\vartheta)} k^{2}+\frac{(1+\vartheta)(3+\vartheta) k}{(5+\vartheta)(11+\vartheta)}-\frac{(3)(3+\vartheta) k}{(5+\vartheta)(11+\vartheta)}
$$

The constants A and B are determined using the conditions of radial stress at inner or outer radius. For four different cases, the above constants are determined and the corresponding equations for radial stresses are established. The derived equations are used to determine the stress distribution at blade mounting location in a steam turbine rotor disc as shown in Fig. 1. The radial stresses are calculated for material properties of $v=0.29, \mathrm{E}=210 \mathrm{GPa}$, $\Upsilon=7810 \mathrm{~kg} / \mathrm{m}^{3}$. To determine the stress distribution along the radial direction, the compatibility criteria is used. The variation of radial stress induced w. r. t. the radius is shown in Fig. 2 with two different cases of considering only the centrifugal force because of rotation of the rotor and considering the blade rotation centrifugal force in the second case. From the variation of radial stress, it can be identified that when the blade rotation centrifugal force is considered, the radial stress increases instantly by almost 4.5 times. The distribution of radial stress in part 4 indicates that there is probability of Mode I crack formation at that blade mounting location. Hence in the next stage, semi elliptical crack is considered at blade mounting location and weight function approach is used to determine the stress intensity factors

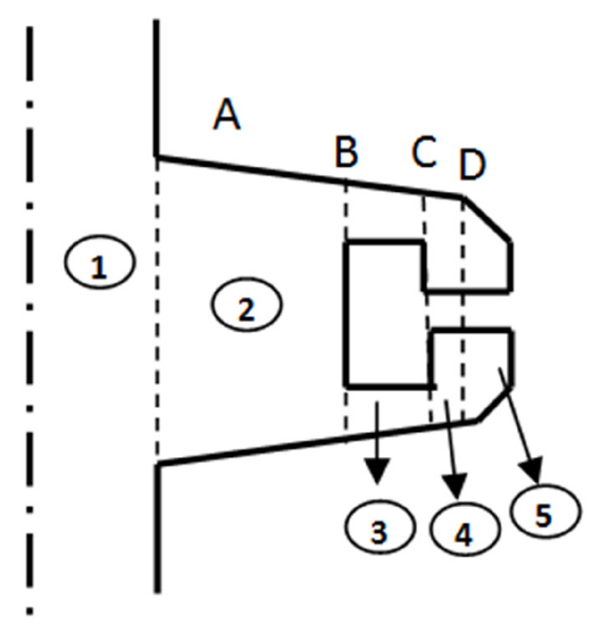

Fig. 1 Blade Mounting Location of turbine rotor

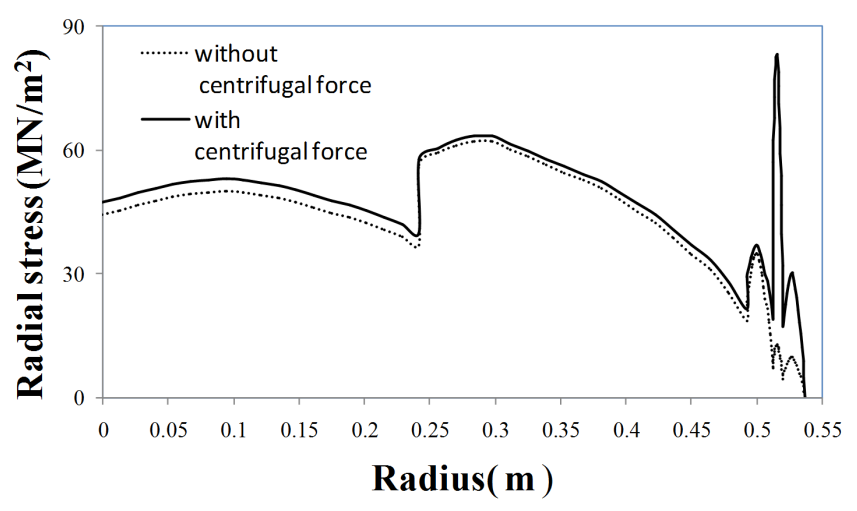

Fig. 2 Distribution of Radial Stress 


\section{Stress Intensity factors using weight function approach}

In this section, a semi elliptical crack Fig. 3 having "2c" as major axis and "a" as the semi minor axis lengths is considered

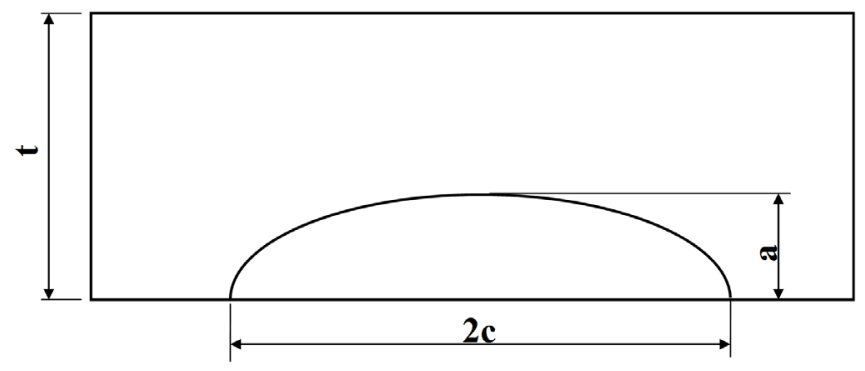

Fig. 3 Semi Elliptical Crack

It is well known fact that the stress intensity factors are highest and lowest at the deepest and surface points of the crack respectively. Hence weight functions are derived for the deepest and lowest points. Once the weight functions are known, the stress intensity factor for the known stress distribution can be determined using Eq. (4). In this equation, the weight function for the deepest point of the crack is given by Eq. (5) and the weight function for the surface point of the crack is given by Eq. (6)

$$
\begin{aligned}
& K_{r}=\int_{0}^{a} \sigma_{r}(x) m(x, a) d x \\
& m_{A}\left(x, a, \frac{a}{c} \cdot \frac{a}{t}\right)=\frac{2}{\sqrt{2 \pi(a-x)}} \\
& {\left[1+M_{1 A}\left(1-\frac{x}{a}\right)^{\frac{1}{2}}+M_{2 A}\left(1-\frac{x}{a}\right)+M_{3 A}\left(1-\frac{x}{a}\right)^{\frac{3}{2}}\right]} \\
& m_{B}\left(x, a, \frac{a}{c} \cdot \frac{a}{t}\right)=\frac{2}{\sqrt{2 \pi x}} \\
& {\left[1+M_{1 A}\left(\frac{x}{a}\right)^{\frac{1}{2}}+M_{2 A}\left(\frac{x}{a}\right)+M_{3 A}\left(\frac{x}{a}\right)^{\frac{3}{2}}\right] .}
\end{aligned}
$$

Now to determine the unknown constants $\mathrm{M}_{1 \mathrm{~A}}, \mathrm{M}_{2 \mathrm{~A}}, \mathrm{M}_{3 \mathrm{~A}}$, $M_{1 B}, M_{2 B}$ and $M_{3 B}$, two reference stress intensity factors with additional third condition is used. The first reference stress intensity factor used is for uniform stress field, the second reference stress intensity factor is for the linearly varying stress field. The third condition is that the second derivative of the weight functions at $\mathrm{x}=0$ must be zero.

By applying the above three conditions, three simultaneous equations are obtained and solving these equations gives the expressions for unknown constants $\mathrm{M}_{1 \mathrm{~A}}, \mathrm{M}_{2 \mathrm{~A}}$ and $\mathrm{M}_{3 \mathrm{~A}}$ are given by Eq. (7). The third condition for the deepest point is that the sum of all the three coefficients should be zero. By applying the above three conditions, three simultaneous equations are obtained and solving these equations gives the expressions for unknown constants $\mathrm{M}_{1 \mathrm{~A}}, \mathrm{M}_{2 \mathrm{~A}}$ and $\mathrm{M}_{3 \mathrm{~A}}$ are given by Eq. (8)

$$
\left.\begin{array}{l}
M_{1 A}=\frac{\pi}{\sqrt{2 Q}}\left(4 Y_{0}-6 Y_{1}\right)-\frac{24}{5} \\
M_{2 A}=3 \\
M_{3 A}=2\left(\frac{\pi}{\sqrt{2 Q}} Y_{0}-M_{1 A}-4\right)
\end{array}\right\}
$$

In Eq. (7) and Eq. (8), the parameters $\mathrm{Y}_{0}, \mathrm{Y}_{1}, \mathrm{~F}_{0}, \mathrm{~F}_{1}$, $\mathrm{Q}$ are taken from Shen et al. [10]

\section{Stress intensity factors for semi elliptical crack at blade mounting location}

As given in the earlier section, there is probability of crack formation at the blade mounting location. So semi elliptical crack is considered as shown in Fig. 4 at blade mounting location. The radial stress distribution obtained at blade mounting locations is used in Eq. (4) and the stress intensity factors are calculated. The stress intensity factor distribution along the crack front for crack depth ratio a/c range of 0.2 to 1 are presented in Tables 1-2, and in Figs. 5-6 for the surface and deepest point respectively.

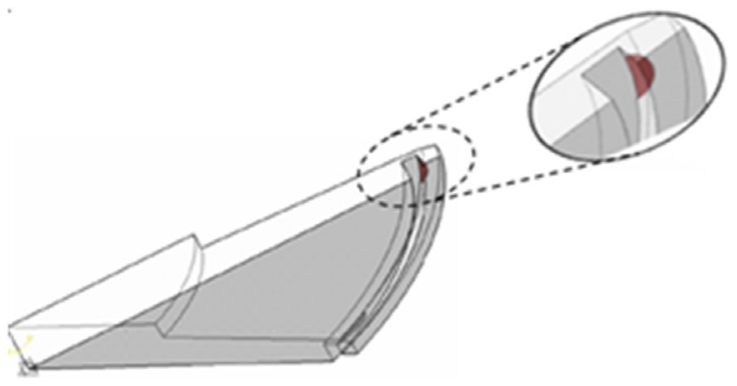

Fig. 4 Semi elliptical crack at blade mounting location

Table 1 Stress Intensity Factor at Surface Point using weight function Method

\begin{tabular}{cccccc}
\hline & \multicolumn{5}{c}{$\mathrm{a} / \mathrm{c}$} \\
\cline { 2 - 6 } & 1 & 0.8 & 0.6 & 0.4 & 0.2 \\
\hline 0.04 & 0.05 & 0.05 & 0.049 & 0.045 & 0.036 \\
0.184 & 0.311 & 0.312 & 0.307 & 0.286 & 0.233 \\
0.328 & 0.447 & 0.454 & 0.451 & 0.428 & 0.359 \\
0.472 & 0.57 & 0.586 & 0.593 & 0.577 & 0.504 \\
0.616 & 0.696 & 0.729 & 0.754 & 0.757 & 0.697 \\
0.76 & 0.834 & 0.892 & 0.948 & 0.989 & 0.973 \\
0.904 & 0.989 & 1.084 & 1.189 & 1.297 & 1.374 \\
1 & 1.104 & 1.232 & 1.384 & 1.558 & 1.738 \\
\hline
\end{tabular}


Table 2 Stress Intensity Factor at deepest Point using weight function Method

\begin{tabular}{cccccc}
\hline & \multicolumn{5}{c}{$\mathrm{a} / \mathrm{c}$} \\
\cline { 2 - 6 } & 1 & 0.8 & 0.6 & 0.4 & 0.2 \\
\hline 0.04 & 0.045 & 0.051 & 0.057 & 0.064 & 0.071 \\
0.184 & 0.280 & 0.317 & 0.358 & 0.406 & 0.458 \\
0.328 & 0.397 & 0.454 & 0.515 & 0.595 & 0.712 \\
0.472 & 0.491 & 0.568 & 0.647 & 0.770 & 0.986 \\
0.616 & 0.568 & 0.667 & 0.763 & 0.933 & 1.270 \\
0.76 & 0.623 & 0.743 & 0.854 & 1.063 & 1.512 \\
0.904 & 0.645 & 0.780 & 0.897 & 1.121 & 1.617 \\
1 & 0.631 & 0.769 & 0.884 & 1.092 & 1.550 \\
\hline
\end{tabular}

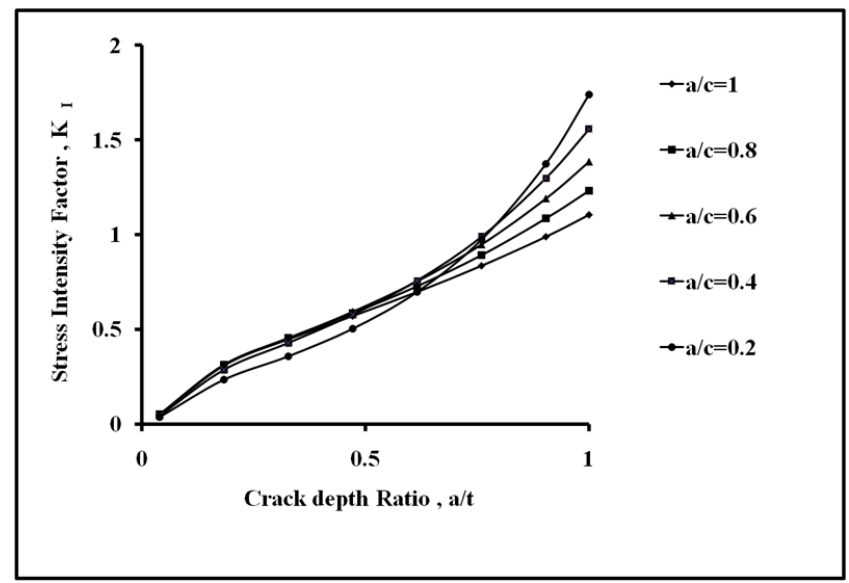

Fig. 5 Stress Intensity Factor at Surface Point using weight function Method

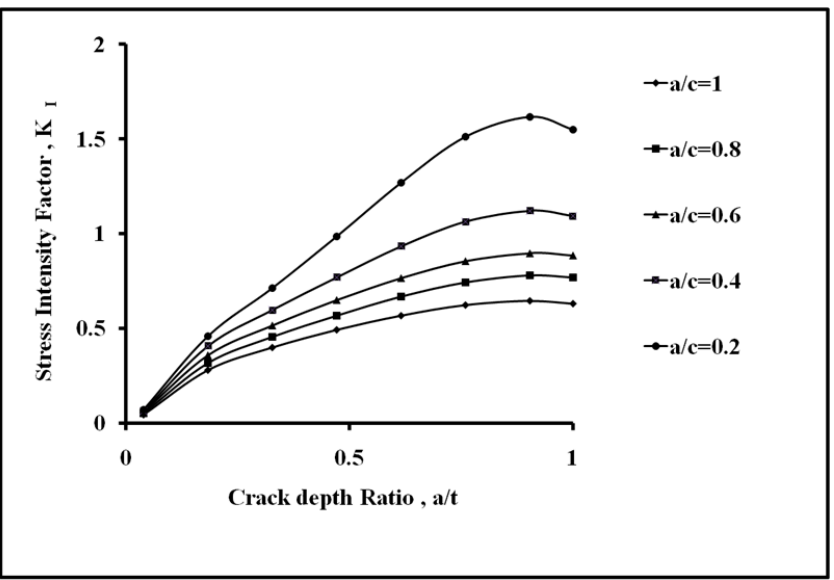

Fig. 6 Stress Intensity Factor at deepest Point using weight function Method

\section{Validation and Discussion}

The stress intensity factors obtained using weight function approach is validated by using the influence coefficient method given by Alegre and Cuesta [5]. The influence coefficient method consists in fitting the stress distribution in the polynomial equation and then determining the stress intensity factor using the Eq. (9)

$$
K_{I}=\sqrt{\frac{\pi a}{Q}}\left[G_{0}\left(A_{0}+A_{p}\right)+G_{1} A_{1}+G_{2} A_{2}+G_{3} A_{3}\right] .
$$

The influence coefficients $\mathrm{G}_{0}, \mathrm{G}_{1}, \mathrm{G}_{2}$ and $\mathrm{G}_{3}$ are calculated using the equations given by Alegre and Cuesta [5]. The stress intensity factor distributions at surface and deepest point are presented in Tables 3-4 and Figs. 7-8. The variation of error is presented in Tables 5-6 and Figs. 9-10 for surface and deepest points respectively. It is observed that the maximum error obtained is $1.2 \%$ and $3 \%$ for the surface points and the deepest point respectively.

Table 3 Stress Intensity Factor at Surface Point using Influence Coefficient Method

\begin{tabular}{cccccc}
\hline \multirow{2}{*}{$\mathrm{a} / \mathrm{t}$} & \multicolumn{5}{c}{$\mathrm{a} / \mathrm{c}$} \\
\cline { 2 - 6 } & 1 & 0.8 & 0.6 & 0.4 & 0.2 \\
\hline 0.04 & 0.049 & 0.049 & 0.048 & 0.044 & 0.035 \\
0.184 & 0.308 & 0.310 & 0.304 & 0.284 & 0.230 \\
0.328 & 0.445 & 0.451 & 0.449 & 0.426 & 0.357 \\
0.472 & 0.568 & 0.584 & 0.591 & 0.575 & 0.502 \\
0.616 & 0.694 & 0.727 & 0.752 & 0.755 & 0.695 \\
0.76 & 0.832 & 0.890 & 0.946 & 0.987 & 0.971 \\
0.904 & 0.987 & 1.082 & 1.188 & 1.295 & 1.373 \\
1 & 1.102 & 1.230 & 1.382 & 1.557 & 1.737 \\
\hline
\end{tabular}

Table 4 Stress Intensity Factor at deepest Point using influence coefficient Method

\begin{tabular}{cccccc}
\hline \multirow{2}{*}{$\mathrm{a} / \mathrm{t}$} & \multicolumn{5}{c}{$\mathrm{a} / \mathrm{c}$} \\
\cline { 2 - 6 } & 1 & 0.8 & 0.6 & 0.4 & 0.2 \\
\hline 0.04 & 0.045 & 0.051 & 0.057 & 0.064 & 0.071 \\
0.184 & 0.275 & 0.312 & 0.353 & 0.4 & 0.453 \\
0.328 & 0.387 & 0.443 & 0.503 & 0.583 & 0.7 \\
0.472 & 0.477 & 0.553 & 0.631 & 0.752 & 0.967 \\
0.616 & 0.552 & 0.649 & 0.745 & 0.912 & 1.247 \\
0.76 & 0.608 & 0.727 & 0.837 & 1.044 & 1.49 \\
0.904 & 0.636 & 0.771 & 0.886 & 1.11 & 1.605 \\
1 & 0.629 & 0.77 & 0.881 & 1.09 & 1.55 \\
\hline
\end{tabular}

Table 5 Error (\%) - Stress Intensity Factor at Surface Point

\begin{tabular}{cccccc}
\hline & \multicolumn{5}{c}{$\mathrm{a} / \mathrm{c}$} \\
\cline { 2 - 6 } & 1 & 0.8 & 0.6 & 0.4 & 0.2 \\
\hline 0.04 & 1.088 & 1.094 & 1.107 & 1.134 & 1.206 \\
0.184 & 0.781 & 0.788 & 0.816 & 0.887 & 1.085 \\
0.328 & 0.479 & 0.482 & 0.499 & 0.547 & 0.674 \\
0.472 & 0.333 & 0.332 & 0.34 & 0.369 & 0.446 \\
0.616 & 0.247 & 0.241 & 0.242 & 0.257 & 0.299 \\
0.76 & 0.198 & 0.188 & 0.183 & 0.187 & 0.205 \\
0.904 & 0.178 & 0.164 & 0.151 & 0.142 & 0.116 \\
1 & 0.178 & 0.16 & 0.14 & 0.114 & 0.024 \\
\hline
\end{tabular}


Table 6 Error (\%) - Stress Intensity Factor at deepest Point

\begin{tabular}{cccccc}
\hline $\mathrm{a} / \mathrm{t}$ & \multicolumn{5}{c}{$\mathrm{a} / \mathrm{c}$} \\
\cline { 2 - 6 } & 1 & 0.8 & 0.6 & 0.4 & 0.2 \\
\hline 0.04 & 0.493 & 0.426 & 0.32 & 0.184 & -0.091 \\
0.184 & 1.825 & 1.666 & 1.529 & 1.344 & 1.164 \\
0.328 & 2.673 & 2.424 & 2.286 & 2.062 & 1.792 \\
0.472 & 3.04 & 2.759 & 2.596 & 2.342 & 1.987 \\
0.616 & 2.953 & 2.705 & 2.502 & 2.235 & 1.875 \\
0.76 & 2.432 & 2.231 & 2.039 & 1.789 & 1.487 \\
0.904 & 1.433 & 1.167 & 1.182 & 0.996 & 0.766 \\
1 & 0.407 & -0.083 & 0.308 & 0.217 & 0.003 \\
\hline
\end{tabular}

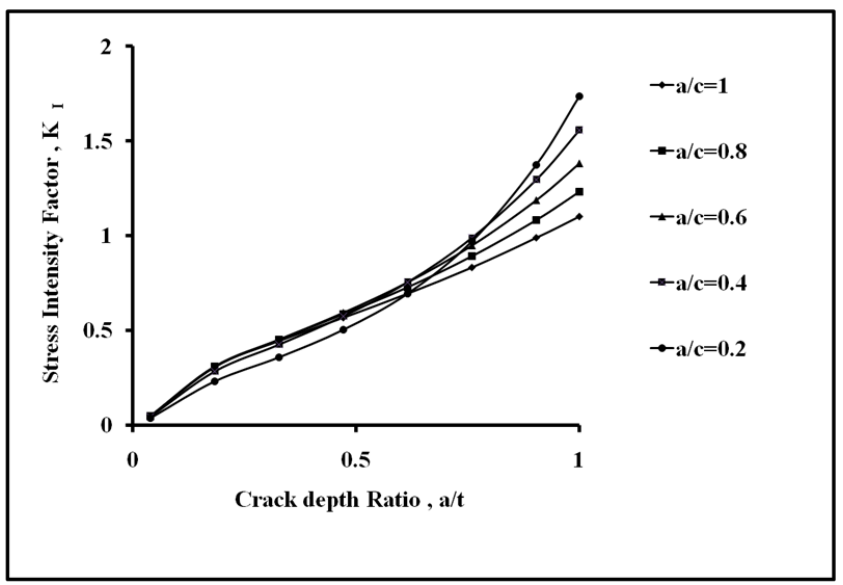

Fig. 7 Stress Intensity Factor at Surface Point using influence coefficient Method

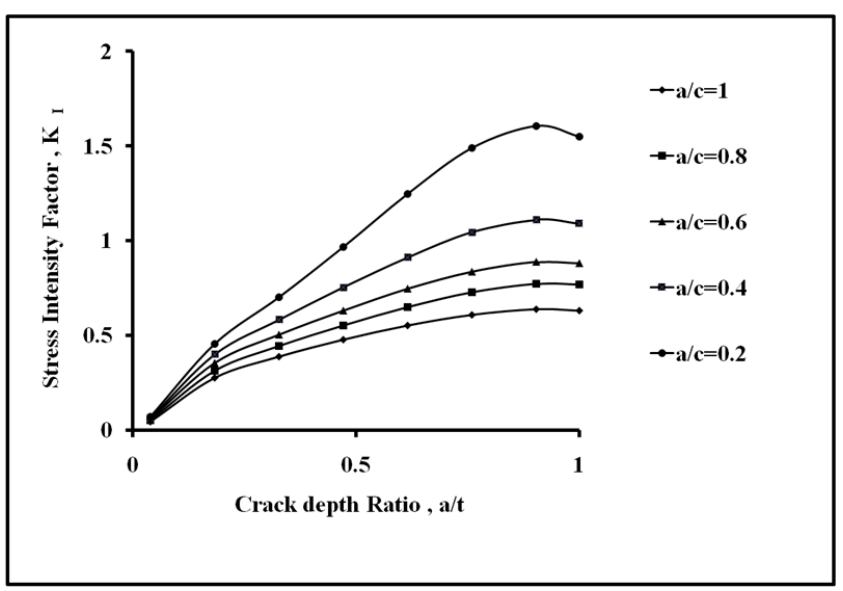

Fig. 8 Stress Intensity Factor at deepest Point using influence coefficient Method

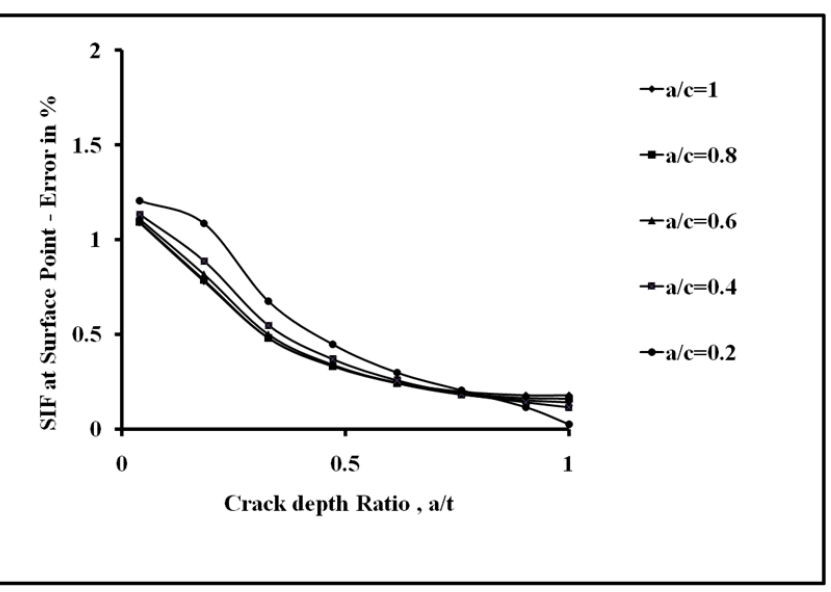

Fig. 9 Error- Stress Intensity Factor at surface Point

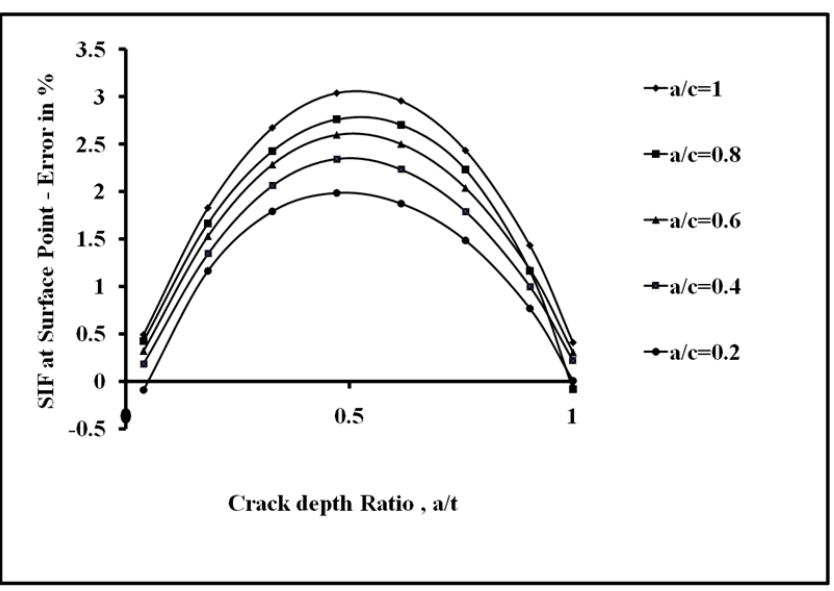

Fig. 10 Error- Stress Intensity Factor at deepest Point

\section{Conclusion}

Equations for radial, tangential stresses and radial displacement for a rotating disc have been derived and applied to a steam turbine rotor disc for determination of these stresses and displacement along the radial direction. It is observed that the radial stress increases suddenly at the blade mounting location which indicates that there is probability of crack initiation and propagation at these locations. A semi elliptical crack is considered and equations for stress intensity factor have been derived using weight function approach. The effect of crack ellipticity $\mathrm{a} / \mathrm{c}$ and crack depth ratio a/t on stress intensity factor was examined. It is observed that the stress intensity factor at surface point increases as the crack depth increases where as for deepest point it marginally decreases as the crack depth ratio almost reaches unity. The results are validated with the influence coefficient approach and the differences were less than $1.2 \%$ for surface point and $3 \%$ for deepest point which are considerable. 


\section{References}

[1] Barella, S., Bellogini, M., Boniardi, M., Cincera, S. "Failure analysis of a steam turbine rotor." Engineering Failure Analysis. 18(6), pp. 15111519. 2011

https://doi.org/10.1016/j.engfailanal.2011.05.006

[2] Hu, D., Wang, R., Fan, J., Shen, X. "Probabilistic damage tolerance analysis on turbine disk through experimental data." Engineering Fracture Mechanics. 87, pp. 73-82. 2012.

https://doi.org/10.1016/j.engfracmech.2012.03.008

[3] Kim, H. "Crack evaluation and subsequent solution of the last stage blade in a low-pressure steam turbine." Engineering Failure Analysis. 18(3), pp. 907-913. 2011.

https://doi.org/10.1016/j.engfailanal.2010.11.004

[4] Han, Q., Chu, F. "The effect of transverse crack upon parametric instability of a rotor-bearing system with an asymmetric disk." Communications in Nonlinear Science and Numerical Simulation. 17(12), pp. 5189-5200. 2012.

https://doi.org/10.1016/j.cnsns.2012.03.037

[5] Alegre, J. M., Cuesta, I. I. "Stress Intensity Factor Equations for Internal Semi-Elliptical Cracks in Pressurized Cylinders." Journal of Pressure Vessel Technology. 133(5), pp. 54501. 2011

https://doi.org/10.1115/1.4002613
[6] Shlyannikov, V. N., Zakharov, A. P., Yarullin, R. R. "Structural integrity assessment of turbine disk on a plastic stress intensity factor basis." International Journal of Fatigue. 92, pp. 234-245. 2016.

https://doi.org/10.1016/j.ijfatigue.2016.07.016

[7] Mazur, Z., Hernandez-Rossette, A. "Steam turbine rotor discs failure evaluation and repair process implementation." Engineering Failure Analysis. 56, pp. 545-554. 2015.

https://doi.org/10.1016/j.engfailanal.2014.10.010

[8] Hou, J., Wescott, R., Attia, M. "Prediction of fatigue crack propagation lives of turbine discs with forging-induced initial cracks." Engineering Fracture Mechanics. 131, pp. 406-418. 2014.

https://doi.org/10.1016/j.engfracmech.2014.08.015

[9] Pang, H. T., Reed, P. A. S. "Microstructure effects on high temperature fatigue crack initiation and short crack growth in turbine disc nickel-base superalloy Udimet 720Li." Materials Science and Engineering A. 448(12), pp. 67-79. 2007.

https://doi.org/10.1016/j.msea.2006.11.016

[10] Shen, G., Glinka, G. "Weight functions for a surface semi-elliptical crack in a finite thickness plate." Theoretical and Applied Fracture Mechanics. 15(3), pp. 247-255. 1991.

https://doi.org/10.1016/0167-8442(91)90023-D 